\title{
REDUÇÃO DE COMPORTAMENTO AUTOLESIVO EM UMA CRIANÇA COM DIAGNÓSTICO DE AUTISMO UTILIZANDO REFORÇAMENTO NÃO CONTINGENTE E TREINO DE MANDO
}

\author{
REDUCTION OF SELF-INJURIOUS BEHAVIOR IN A CHILD WITH AUTISM \\ DIAGNOSIS USING NON-CONTINGENT REINFORCEMENT AND MAND TRAINING
}

MARCUS ViníCIUS FonseCA DE GARCia

THAIS PORLAN OLIVEIRA

Universidade Federal de Minas Gerais, BRASIL E

InStituto NACIONAL de CIÊNCIA E TECNOLOGIA SOBRE COMPORTAMENTO, COGNIÇÃo E ENSINO, BRASIL

\begin{abstract}
RESUMO
O estudo buscou verificar a efetividade de um tratamento planejado com base em análise funcional experimental, visando reduzir o comportamento autolesivo [SIB] de um adolescente de 13 anos com transtorno do espectro de autismo, associado a deficiência intelectual, atendido em uma instituição brasileira. $\mathrm{O}$ procedimento envolveu expor o participante alternadamente a quatro condições que poderiam estabelecer ocasiões para a ocorrência de SIB, com registro da frequiência de respostas autolesivas. A intervenção foi realizada a partir da identificação da manutenção do comportamento por fuga. Foram planejados alguns procedimentos específicos destacando o reforçamento não contingente [NCR] e treino de mando no ambiente escolar, obtendo-se significativa redução da frequência de SIB. Conclui-se que procedimentos implementados a partir de análise funcional experimental mostram-se efetivos para a redução de SIB.
\end{abstract}

Palavras-chave: comportamento autolesivo, autismo, análise funcional experimental, reforçamento não contingente, treino de mando.

The study aimed to verify the effectiveness of a treatment based on experimental functional analysis in order to reduce the self-injurious behavior [ SIB ] in a 13 year old adolescent with autism spectrum disorder associated with intellectual disability, who attended a Brazilian institution. The experimental functional analysis procedure involved exposing the participant alternately to four conditions that could establish occasions for the occurrence of SIB, with the record of frequency of self-injurious responses. The intervention was developed based on the identification that the participant's SIB was maintained by escape from demand. The intervention involved some specific non-contingent reinforcement [NCR] procedures, and mand training at school. Experimental functional analysis procedures showed to be effective in identifying SIB function, and in fostering its reduction through appropriate procedures.

Key words: self-injurious behavior, autism spectrum disorder, experimental functional analysis, noncontingent reinforcement, mand training.

\footnotetext{
Este trabalho é parte da dissertação de mestrado do primeiro autor, desenvolvida junto ao Programa de Pós-Graduação em Psicologia da UFMG. Agradecimentos aos componentes da banca examinadora: Profa. Camila Graciella Santos Gomes; Prof. Marcelo Frota Lobato Benvenuti e Profa. Viviane Verdu Rico, aos funcionários da APAE/BH e aos estagiários Fábio Henrique Albuquerque Corrêa, Rafael da Costa Silva e Xilander Rocha Resende. Financiamento: Bolsa de mestrado da CAPES para o primeiro autor. Apoio: Instituto Nacional de Ciência e Tecnologia sobre Comportamento, Cognição e Ensino - INCT-ECCE, por meio da FAPESP (Processo 2008/57705-8) e do CNPq (Processo 573972/2008-7).
} 
O comportamento autolesivo (SIB, do inglês SelfInjurious Behavior) é um problema muitas vezes crônico, que pode trazer sérios riscos à integridade física do indivíduo e grandes prejuízos para seu desenvolvimento e qualidade de vida. O SIB pode ser definido como classes de respostas que produzem danos físicos ao próprio corpo (Tate \& Baroff, 1966). Topografias de comportamentos autolesivos observadas em pessoas com desenvolvimento atípico são variadas e podem envolver bater a própria cabeça contra superfícies ou objetos, dar tapas ou socos no próprio rosto ou cabeça, morder a si próprio, abocanhar a mão, cutucar a garganta ou os olhos, puxar ou arrancar o próprio cabelo, ferir-se com as unhas, ingerir objetos não comestíveis, dentre outras (Carr, 1977; Matson \& LoVullo, 2008).

A incidência e persistência de SIB na população de pessoas com deficiência severa são altas e requerem investigações detalhadas sobre avaliação e tratamento. Kahng, Iwata e Lewin (2002) consideraram que entre $10 \%$ a $14 \%$ dos indivíduos com deficiência intelectual exibem SIB, dos quais a incidência é de $71,2 \%$ para casos de deficiência severa/profunda. Dentre os diagnósticos secundários, foi apontada a incidência de SIB em 7\% dos casos de síndrome de Rett, $10,1 \%$ dos casos de transtornos do espectro do autismo e $20 \%$ dos casos de deficiência sensorial visual e/ou auditiva (Kahng et al., 2002).

Entre os fatores de risco para o SIB, foram encontrados outros aspectos, além dos tipos e graus de deficiências, tais como idade cronológica associada à condição perinatal (Baghdadli, Pascal, Grisi, \& Aussilloux, 2003). Uma variável considerada altamente preditiva ao risco de SIB é a baixa habilidade para realização de atividades diárias, com destaque para habilidades verbais restritas (Baghdadli et al., 2003; Saloviita, 2000). Assim sendo, programas de intervenção que envolvam ensino, em alguma medida, de habilidades verbais para facilitar a comunicação, configuram-se como hipoteticamente importantes para redução de SIB. A literatura destaca que alguns indivíduos, em função de déficits de habilidades comunicativas em seu repertório, podem se engajar em comportamentos auto-prejudiciais e, por meio deles, produzir acesso a consequências reforçadoras (Chambers \& Rehfeldt, 2003; Frea, Arnold, \& Vittimberga, 2001; Kelley, Shillingsburg, Castro, Adisson, \& LaRue, 2007).

É importante que se objetive a aprendizagem de comportamentos que possam vir a produzir conseqüências reforçadoras e relevantes em situações naturais, em concorrência com o SIB. De acordo com a definição de Skinner (1957), mando é o operante verbal que especifica, sob controle de condições antecedentes do falante, reforçadores que outros podem lhe fornecer. Emitir um mando é, portanto, um comportamento que tem como consequência a possibilidade de trazer benefício direto ao falante (Barros, 2003; Rosales \& Rehfeldt, 2007). Ensinar a emitir mandos é importante porque crianças aprendem a comunicar consequências específicas que esperam em determinado contexto, o que possibilita maior controle social sobre seu ambiente, ou seja, permite o engajamento em relacionamentos nos quais seus comportamentos produzem mudanças nos comportamentos de outros (Murphy, D. Barnes-Holmes, \& Y. Barnes-Holmes, 2005; Troconis, 2011; Gutierrez Jr. et al., 2007).

Para subsidiar o planejamento de intervenções como o treino de mando, é preciso que se identifique a função do comportamento problema. Iwata, Dorsey, Slifer, Bauman e Richman (1982/1994) descreveram uma metodologia de análise funcional experimental que envolvia a manipulação sistemática de variáveis ambientais antecedentes e consequentes sob diferentes condições controladas, viabilizando a identificação de consequências mantenedoras do SIB. Esse trabalho impulsionou uma mudança de direcionamento nos contextos de aplicação, a partir da utilização sistemática de delineamentos experimentais em avaliações préintervenção, cujos resultados garantiram maior confiabilidade a planejamentos de diversas modalidades individualizadas de tratamento, baseadas na função do SIB, e não apenas em sua topografia. O desenvolvimento de análises do SIB baseadas em controle experimental aperfeiçoou tecnologias de avaliação que se baseavam em métodos indiretos, como entrevistas com pais e/ou cuidadores, e em métodos descritivos, como a observação direta e registro sistemático (Lerman \& Iwata, 1993).

As quatro condições básicas de manejo de variáveis utilizadas na análise funcional experimental, no grupo liderado por Iwata, são denominadas: (a) sozinho, (b) atenção, (c) brincadeira e (d) demanda (Iwata et al., 1982/1994). Na condição sozinho, a pessoa permanece sozinha em uma sala, sem nenhuma interação social. Essa condição é utilizada para identificar reforçamento automático, caso a frequência de SIB permaneça alta. Na condição atenção, há reforçadores disponíveis e atenção social é brevemente fornecida, apenas imediatamente após a ocorrência de SIB. Na condição brincadeira, que é considerada controle para as demais condições, reforçadores estão disponíveis e são mantidas apenas interações sociais amenas, caracterizadas por brincadeiras. Espera-se, por exemplo, que o SIB não ocorra nessa condição, caso seja mantido por atenção, uma vez que a pessoa terá atenção abundante disponível. A frequência alta de SIB na condição brincadeira, associada à frequência alta também nas demais condições, pode sugerir que o SIB é mantido por reforçamento automático. $\mathrm{Na}$ condição demanda, são apresentadas demandas para realização de atividades, especialmente aquelas identificadas previamente como aversivas para a pessoa, e qualquer ocorrência de SIB é consequenciada imediatamente pela cessação da demanda.

Tecnologias para tratamento a partir da análise funcional experimental configuram o planejamento de intervenções baseadas em evidências para pessoas com transtornos do espectro do autismo, sendo prescritas por instituições internacionalmente reconhecidas, como parte indispensável do tratamento (Broadstock \& Lethaby, 2008; Sturmey \& Hersen, 2012). No Brasil, entretanto, estudos que atestem a aplicação de procedimentos experimentais de avaliação e tratamento do SIB são escassos, ao contrário dos Estados Unidos, onde, apenas em um periódico, o Journal of Applied Behavior Analysis 
(JABA), foram publicados 322 artigos sobre o SIB, entre 1968 e 2010 (Garcia \& Gomes, 2012).

Ceppi e Benvenuti (2011) realizaram uma revisão bibliográfica de estudos empíricos que descreviam procedimentos de tratamento baseados em avaliação funcional pré-intervenção em casos de SIB, publicados entre os anos de 1991 e 2010. Foram selecionando 46 estudos, nenhum deles brasileiro, e descritos os tratamentos específicos que foram utilizados em 25 desses estudos. Seus resultados mostraram uma ampla gama de possibilidades de tratamento analítico-comportamental com base na função do SIB, ressaltando-se, dentre eles, o reforçamento não-contingente [Non-contingent reinforcement - NCR], reforçamento diferencial de comportamento alternativo [differential reinforcement of alternative behavior - DRA] e reforçamento diferencial de outro comportamento [differential reinforcement of other behavior - DRO]. Todas são formas tradicionais de intervenção que envolvem manejos específicos de reforçadores. No NCR, reforçadores são disponibilizados em esquemas de tempo (independentes de comportamento); no DRA, respostas específicas são consequenciadas, enquanto o SIB não produz reforço; no DRO, quaisquer outros comportamentos são consequenciados em períodos em que o SIB não ocorre.

Dentre os procedimentos para redução de SIB, o NCR destaca-se por viabilizar o acesso a reforçadores independente da resposta do indivíduo, em esquemas de tempo, o que, de acordo com Carr et al. (2000), é uma vantagem por enfraquecer a relação entre resposta-reforço, pela garantia de taxas consistentes de reforçamento. Outras vantagens discutidas sobre o procedimento envolvem o fato de ser de fácil implementação, por exigir controle de tempo, não demandar atenção direta aos comportamentos problema e produzir menos efeitos secundários, como o aumento da frequência inicial do comportamento, que ocorre no caso da extinção (Carr et al., 2000; Lalli, Casy, \& Kate, 1997).

Tendo em vista a gravidade do SIB, é evidente a importância da implementação de procedimentos de avaliação e tratamento efetivos. O presente estudo teve por objetivo verificar a efetividade de procedimentos de reforçamento não contingente e treino de mando planejados com base nos resultados de uma análise funcional experimental, visando à redução do SIB em um caso de transtorno do espectro do autismo. A realização da pesquisa em uma instituição brasileira constitui uma contribuição para o já estabelecido corpo de evidências que embasa a adoção de procedimentos de análise funcional experimental para o planejamento de tratamentos em casos de SIB.

\section{Participante}

\section{MÉTODO}

O participante foi selecionado após contato inicial com profissionais da instituição e análises de prontuários. O critério principal foi a escolha de um participante que, embora tivesse uma deficiência severa, exibisse uma topografia de SIB que não representasse sérios riscos de ferimentos imediatos. Foi selecionado um adolescente (L) morador de uma casa-lar mantida pela APAE de Belo Horizonte, 13 anos de idade, com severo atraso no desenvolvimento neuropsicomotor e déficit visual. L. não apresentava fala e interagia muito pouco com outras pessoas. O participante começou a andar aos seis anos, sendo dependente de cuidadores para todas as atividades de vida diária. L. recebia ajuda para levar alimento à boca e usava fraldas. Em relatório médico, encontraram-se os diagnósticos de retardo mental grave (CID10 - F72) e autismo infantil (CID10 - F84.0). Trata-se de um caso de transtorno do espectro do autismo de nível 3, de acordo com os especificadores de gravidade do DSM-5 (Associação Psiquiátrica Americana, 2013).

L. foi abrigado na APAE em setembro de 2011, após a guarda ser retirada da mãe, devido a histórico de dependência química, negligência e maus tratos. De acordo com o prontuário, o garoto encontrava-se desnutrido e há relato de "auto-agressividade constante" nessa época. L. tem histórico de internações psiquiátricas devido a "crises nervosas" com auto-agressão e gritos, sendo que a última dessas internações ocorreu em dezembro de 2012. Em um relatório multidisciplinar de 2013, há a observação de que as auto-agressões de L. ocorriam quando ele queria "comunicar algo", embora existam vários outros registros de prontuário que mencionavam crises de auto-agressividade sem causas ou contextos específicos. Em um relatório da escola que L. frequentava aos nove anos, consta que batia no rosto e ficava muito nervoso "sem causa aparente". De acordo com os documentos consultados, as crises de autoagressão eram sempre sucedidas por solicitações de reavaliação da medicação psiquiátrica, o que era feito, mas sem que se produzisse redução do SIB. Há observações de que visitas da mãe biológica e o posterior afastamento desta causariam sofrimento ao garoto, aumentando, assim, as auto-agressões. A pesquisa seguiu todas as normas requeridas pelo Comitê de Ética em Pesquisa da UFMG, segundo a Resolução CNS/466 (CAAE 36660714.1.0000.5149).

\section{Procedimentos \\ Medida.}

A topografia específica de respostas de SIB emitidas por L., considerada neste estudo, foi definida como tapas de leve/moderada intensidade direcionados à cabeça ou rosto, com qualquer uma das mãos. O potencial lesivo imediato do comportamento geralmente não passava de um leve rubor facial, cujas implicações no longo prazo, no entanto, podem incluir o risco de lesões neurológicas, devido ao caráter repetitivo e crônico do comportamento.

\section{Linha de base no ambiente doméstico.}

Foram realizados seis períodos de observação direta, com duração de 40 minutos cada um, em dias diferentes da semana, com registros videogravados no ambiente doméstico. As videogravações foram assistidas logo após cada dia de filmagem e não foram observadas ocorrências de respostas autolesivas no ambiente doméstico. 
No ambiente doméstico, L. ficava sob os cuidados de uma mãe social, funcionária que exerce o papel de cuidadora dos sete moradores da casa-lar, com mais duas auxiliares. Durante essas observações, L. permanecia à vontade, deitado no chão do quintal ou no sofá da sala, sem que lhe fosse apresentado qualquer tipo de demanda. A casa-lar é ampla com quatro quartos, dois banheiros e um quintal, ao qual os moradores tem acesso contínuo. Durante os períodos de observação direta, L. permanecia sozinho a maior parte do tempo, mas a mãe social mantinha interação verbal intermitente, dizendo seu nome e, em alguns momentos, estabelecendo contato físico com carinhos na cabeça e nas costas. L. sorria nesses momentos. Nos horários em que foram realizadas as observações, estavam presentes apenas o pesquisador, a mãe social e uma auxiliar, pois os demais moradores estavam em horário escolar.

\section{Linha de Base no ambiente escolar.}

Para registros de linha de base no ambiente escolar, foram realizados três períodos de observação direta, com duração de 40 minutos cada um, em dois dias diferentes da semana no período da manhã, com registros videogravados. $\mathrm{Na}$ escola, o participante frequentava cinco vezes por semana, durante quatro horas diárias, uma sala estruturada do Programa TEACCH, onde realizava atividades descritas como de nível 1, que envolvem transferências de objetos, realizadas com apoio físico e verbal. A turma tinha uma professora e uma auxiliar e apenas três alunos, todos com deficiência severa e necessidade de nível alto de apoio. Eram conduzidos períodos breves de cerca de quatro minutos de atividades em uma mesa específica, intercalados com períodos em que o participante permanecia apenas sentado em uma cadeira posicionada em uma área denominada "lazer", onde não eram apresentadas demandas de realização de atividades.

L. permanecia sentado na cadeira "lazer" por períodos mais longos, de cerca de quinze minutos em média, enquanto atividades eram conduzidas com outros alunos. Eram utilizados objetos, descritos no programa pedagógico como referências, que sinalizavam idas para a mesa de atividade. Tais objetos eram: uma pequena peça de madeira vermelha, de 3x3 $\mathrm{cm}$, sinalizando a ida para a mesa de atividades; uma pequena almofada de $5 \times 5 \mathrm{~cm}$, sinalizando a ida para a cadeira do lazer; um mouse de computador, para a atividade de informática (conduzida por outra professora, em outra sala); uma placa de $8 \times 6 \mathrm{~cm}$ com grãos de feijão e arroz afixados com fita adesiva, que sinalizavam a ida para o refeitório. Não eram utilizadas consequências tangíveis que poderiam ter função de reforçadores durante a realização de atividades.

As videogravações da linha de base na escola foram assistidas logo após cada dia de filmagem, e foi registrada a frequência de ocorrência das respostas autolesivas. A frequência das respostas alvo de SIB nas observações diretas no ambiente escolar foi utilizada como linha de base para a intervenção, realizada apenas na escola, tendo seu planejamento baseado nos dados da análise funcional experimental.

Análise funcional experimental.

Após os registros de linha de base, o procedimento de análise funcional experimental envolveu expor o participante alternadamente a quatro condições que poderiam estabelecer ocasiões para a ocorrência de SIB, com o registro da frequência de respostas autolesivas em cada uma das condições. As quatro condições foram as descritas na introdução: sozinho, atenção, brincadeira e demanda (Iwata et al., 1982/1994).

Foram conduzidas 20 sessões de análise funcional experimental, com duração de 10 minutos cada. As sessões foram realizadas em dois dias diferentes da semana (doze sessões em um dia e oito sessões no outro), com um intervalo de uma semana entre cada dia, e foram videogravadas. As quatro condições experimentais foram manipuladas em sequência alternada. Os ambientes físicos e pessoas presentes foram os mesmos nas primeiras doze sessões, mas foram modificados, conforme descrito no item ambiente experimental, nas oito sessões seguintes. $\mathrm{O}$ pesquisador realizou duas reuniões com a equipe de profissionais que participou das coletas, para explicar os procedimentos, de acordo com as especificações sugeridas por Iwata et al. (2000).

\section{Ambiente experimental}

$\mathrm{O}$ primeiro autor coordenou o andamento das sessões, fornecendo dicas no momento da condução das sessões pela equipe, e realizou as filmagens. As primeiras doze sessões foram conduzidas pelo pesquisador, por um estagiário de psicologia, dois psicólogos e uma fonoaudióloga. O estagiário de psicologia conduziu as sessões atenção, o psicólogo da APAE conduziu as sessões demanda, a psicóloga da APAE conduziu as sessões brincadeira e a fonoaudióloga realizou a cronometragem durante as sessões, indicando os momentos de início e de término das interações com o participante. A duração total das doze sessões iniciais foi de três horas.

Devido a especificidades do contexto institucional, não foi possível conduzir todas as sessões de avaliação no mesmo ambiente físico e foram feitas adaptações para a realização dos procedimentos no segundo dia de coleta na Clínica Intervir, da APAE/BH. Participaram dessas oito últimas sessões o pesquisador, o estagiário de psicologia e uma terapeuta ocupacional da APAE. O pesquisador atuou como terapeuta nas sessões atenção, o estagiário atuou como terapeuta nas sessões demanda e a terapeuta ocupacional atuou como terapeuta nas sessões brincadeira, enquanto o pesquisador e o estagiário se revezaram na realização das filmagens. As oito sessões, nesse segundo dia, levaram duas horas e quarenta minutos para serem concluídas.

As salas onde foram realizadas as sessões variaram de um dia para o outro de coleta, devido à mudança de ambiente. De maneira geral, as dimensões das salas nas quais foram realizadas as sessões sozinho foram menores $(3,8 \times 5,9 \mathrm{~m}$ e $3 \times 4,1 \mathrm{~m})$ e maiores nas demais condições $(7,7 \times 5 \mathrm{~m}$ e $4,2 \times 3 \mathrm{~m})$. Na condição sozinho, a câmera ficava posicionada em uma janela, de modo a focalizar o participante, sem que houvesse qualquer contato com o experimentador. $\mathrm{Na}$ condição demanda, havia na sala uma mesa com duas cadeiras, uma de frente para a outra, para onde o participante era direcionado pelo terapeuta e as demandas eram apresentadas. 
Tabela 1

Características de Cada uma das Quatro Condições Experimentais Manipuladas na Fase de Análise Funcional Experimental.

Condição Ambiente físico e social $\quad$ Procedimentos

Sozinho

Sala vazia, sem a presença de materiais/objetos

$\begin{array}{ll} & \text { Mobiliários (um sofá, } \\ \text { Atenção } & \text { uma televisão, uma cama } \\ & \text { e um colchonete), } \\ & \text { livremente disponíveis }\end{array}$

Brincadeira

Um colchonete, livremente disponível
Nenhum contato social com o participante, nem demandas ou atividades propostas.

Sessões iniciam com o terapeuta declarando que tinha que limpar a sala e não poderia atender o participante. Em seguida, a cada ocorrência de um comportamento-alvo, o terapeuta se aproximava do participante e falava para ele parar, pois poderia se machucar. Tal frase era pareada com contato físico por cinco segundos, durante os quais o terapeuta tocava a cabeça do participante.

Em intervalos de 30 segundos, o terapeuta respondia a qualquer comportamento social apropriado iniciado pelo participante. $\mathrm{O}$ colchonete no chão foi utilizado para as interações entre a terapeuta e o participante, com músicas cantadas e leves toques carinhosos do terapeuta a cada aproximação do participante.

Mesa, duas cadeiras e materiais para atividades de encaixe de peças
O terapeuta iniciou as sessões dizendo "atividade" e pediu que o participante tirasse e colocasse as meias ou que realizasse atividades de encaixe de objetos. Após cinco segundos, caso o participante não atendesse à solicitação, o terapeuta demonstrava a resposta correta ou fornecia uma dica física. Após a dica, caso o participante não atendesse à solicitação, o terapeuta o guiava fisicamente para realizar a tarefa. Instruções foram repetidas até que a sessão terminasse, sem nenhum outro tipo de interação entre terapeuta e participante. A cada ocorrência do comportamentoalvo, a instrução era imediatamente terminada sem comentários, e a próxima tentativa era adiada por 40 segundos.

\section{Condições experimentais}

São descritas na Tabela 1 as características de cada uma das quatro condições experimentais manipuladas (ver também a descrição na introdução).

\section{Concordância entre observadores sobre a análise funcional}

A concordância entre observadores para as sessões de análise funcional experimental foi calculada por meio do método de concordância total. Um observador independente assistiu a cinco minutos de cada sessão videogravada $(50 \%)$ e registrou as respostas de SIB. Os escores totais de concordância foram calculados somandose as respostas de SIB observadas pelo observador independente e pelo pesquisador. $\mathrm{O}$ número menor foi dividido pelo número maior e o quociente foi multiplicado por 100 . A média de concordância foi de $98,5 \%$ (variação entre $96,7 \%$ e $100 \%$ ).

\section{Intervenção}

As alterações nas contingências foram realizadas em fases, segundo a descrição a seguir, em um delineamento de quasi-reversão.
Fase 1 - Linha de base;

Fase 2 - Participante permaneceu em sala com livre acesso a um colchonete durante o recreio, não indo para o pátio, como ocorria anteriormente;

Fase 3 - Além da permanência em sala, com acesso ao colchonete durante o período do recreio, foi também introduzido o uso de reforçador comestível, durante a realização de atividades;

Fase 4 - Reversão à linha de base;

Fase 5 - Reintrodução do recreio em sala, com livre acesso ao colchonete;

Fase 6 - Continuidade do recreio em sala durante o recreio, com livre acesso ao colchonete e realização de uma revisão curricular, sendo substituídas as atividades de transferência de objetos por um procedimento de treino de mando;

Fase 7 - Acrescido a permanecer em sala durante o recreio e à realização do treino de mando, foi também utilizado um procedimento de NCR (reforçamento nãocontingente).

Treino de mando.

No treino de mando, o participante foi ensinado a solicitar o acesso a reforçadores por meio de toques na 
mão da professora. Os reforçadores foram comestíveis: maçã picada sem casca, uva sem semente e banana picada. A escolha de frutas como reforçadores ocorreu em função de observações do participante no refeitório, em situações naturais. Foi dada prioridade ao uso de frutas como reforçadores devido não apenas à preferência, mas também considerando o valor nutricional desses alimentos. Nas primeiras sessões de treino de mando, foi utilizado apenas o acesso à maçã. $\mathrm{O}$ uso de reforçadores variados (maçã, uva e banana) foi iniciado a partir da sexta sessão de treino de mando, sendo sempre dada prioridade àquele que produzia, no momento, maior número de respostas independentes. A maçã foi também fornecida sempre que o participante chegava à mesa de atividades, quando imediatamente lhe era fornecido um pequeno pedaço da fruta, bem como logo após ele se sentar na cadeira do lazer.

Para a inclusão dos alimentos como reforçadores no treino de mando, foram dadas orientações à professora $\mathrm{e}$ à auxiliar em relação ao uso de dicas físicas, visuais e verbais (prompts), e sobre como introduzir a redução gradual dessas dicas, em um processo de esvanecimento (fading out), um procedimento que envolvia treino por tentativas discretas.

A professora foi instruída a posicionar uma das mãos sobre a mesa de atividades e perguntar ao participante se ele gostaria de pedir um pedaço de maçã. A auxiliar deveria direcionar a mão do participante e posicioná-la sobre a mão da professora, que imediatamente, com a outra mão, forneceria um pequeno pedaço de maçã. A fim de garantir que o participante respondesse sob controle da mão da professora posicionada sobre a mesa e não sob controle de um local específico da mesa, a professora variava a posição da sua mão a cada tentativa discreta. Foi importante que a maçã fosse fornecida com a outra mão, para que o participante não tentasse buscar a maçã na mão posicionada sobre a mesa, mas sim que produzisse o acesso imediato à maçã ao apenas tocar levemente a mão da professora sem a maçã visível. A professora foi instruída também a atrasar o reforço e iniciar nova tentativa se algum SIB ocorresse logo após a resposta de mando, de modo que o SIB não fosse sucedido imediatamente por reforço positivo. Respostas independentes de mando, ocorridas sem nenhuma dica física, foram registradas ao longo das sessões.

\section{Reforçamento não-contingente (NCR)}

Este procedimento consistia no fornecimento de reforçadores comestíveis (frutas) e sociais (brincadeiras com falas exclamativas) em um esquema de tempo variável (VT), que variou entre um e três minutos, nos períodos em que o participante permanecia na cadeira do lazer, enquanto a professora realizava atividades com outros alunos. A professora e a auxiliar foram instruídas a fornecer reforçadores comestíveis ao participante enquanto este se encontrava na cadeira do lazer.

Registro

Durante as sete fases foram realizados 41 registros videogravados, com duração de 40 minutos cada um, de sessões de intervenção. Os registros foram feitos nos 10 minutos finais do recreio e 30 minutos subsequentes em sala, durante períodos de atividades, intercalados com a permanência na cadeira do lazer.

\section{Concordância entre observadores na fase de intervenção}

Para as sessões de intervenção, o cálculo para a observação do SIB foi realizado da mesma maneira que para as sessões de análise funcional, porém o observador independente assistiu a $25 \%$ de cada uma das sessões videogravadas. A média de concordância foi de $96,6 \%$ (variação entre $66,7 \%$ e $100 \%$ ). A concordância entre observadores para as respostas independentes de mando também foi calculada por meio do mesmo método. $\mathrm{O}$ observador independente assistiu a todas as sessões de treino de mando videogravadas e registrou as respostas consideradas independentes. A média de concordância neste caso foi de $99,3 \%$ (variação entre $75 \%$ e $100 \%$ ).

\section{Linha de base}

\section{RESULTADOS}

Nas sessões de linha de base no ambiente escolar, L. apresentou o comportamento alvo em variados momentos e frequentemente se jogava ao chão, quando era levado à mesa de atividades. Foi possível também a observação de outras variáveis relevantes: ocorriam muito poucas consequências sociais, como elogios exclamativos após a realização de atividades; o Programa TEACCH parecia ser aplicado de modo rígido, por meio do seguimento de regras, aparentemente sem conhecimento aprofundado para aplicação de estratégias analíticocomportamentais, o que parecia tornar o modelo pouco eficaz para o ensino.

\section{Análise funcional experimental}

Os resultados da análise funcional experimental foram representados na Figura 1 por meio da média de respostas de SIB (R/min) apresentadas por sessão. Os dados sugeriram que o SIB do participante era socialmente mediado, mantido por reforçamento negativo (fuga de demanda). O SIB permaneceu com uma frequência alta em todas as sessões demanda, observando-se estabilidade da frequência de SIB, sobretudo nas três últimas sessões da condição. Na primeira série de quatro sessões, não houve qualquer ocorrência de SIB nas sessões sozinho, atenção e brincadeira. Na primeira sessão demanda, a frequência de SIB foi alta, retornando a zero nas sessões seguintes (sozinho, atenção e brincadeira) e aumentando novamente, logo na próxima sessão demanda.

Ocorreu um aumento da frequência de SIB na sessão 14 (atenção). $\mathrm{O}$ aumento especificamente observado nessa sessão pode ser atribuído a adaptações realizadas no ambiente físico de avaliação. Tratava-se da primeira sessão atenção no ambiente da Clínica Intervir. Desse modo, foi improvisado um espaço limitado em um amplo salão da clínica, espaço este cercado por colchonetes. Ao limitar o espaço do amplo salão com os colchonetes, por equívoco, não foi disponibilizado um colchonete no chão para o participante, como havia ocorrido nas sessões atenção anteriores. 


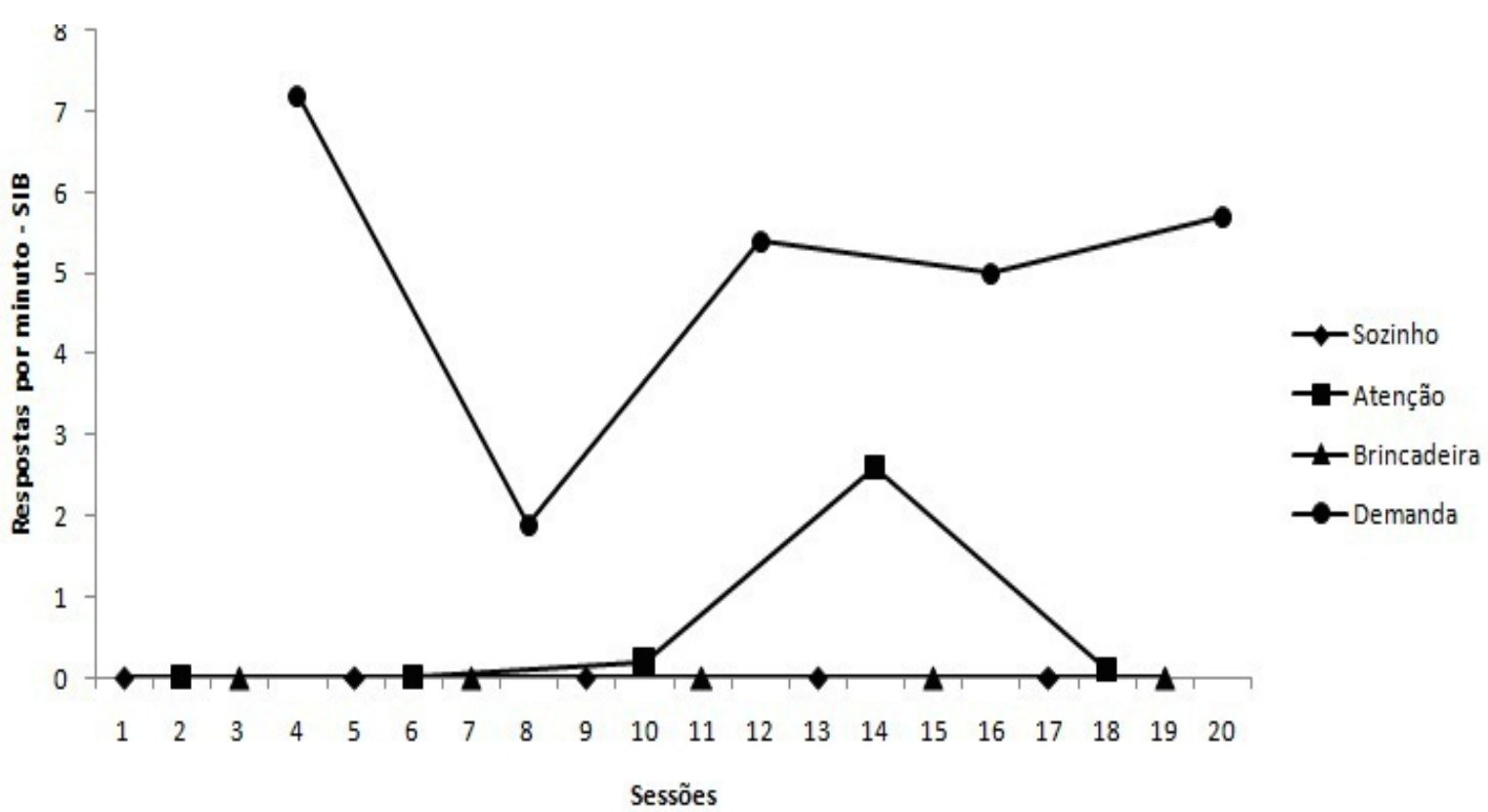

Figura 1. Análise funcional experimental: taxa média de respostas de SIB (R/min) ao longo das sessões, como função das condições (Sozinho, Atenção, Brincadeira e Demanda).

O aumento do SIB na sessão 14 pode ser interpretado, assim, como possíveis respostas à aversividade de um contexto pobre em reforçadores. $\mathrm{Na}$ sessão atenção seguinte (sessão 18), o colchonete foi posicionado no chão, sendo logo buscado pelo participante, que nele se deitou e permaneceu por toda a sessão.

\section{Intervenção}

Recreio em sala de aula, treino de mando e reforçamento não-contingente.

Como mostra a Figura 2, foi observada uma rápida redução do SIB já na primeira fase da intervenção, na qual o participante permaneceu em sala durante o recreio, com livre acesso a um colchonete. Após a introdução do reforçador comestível durante a realização de atividades, que ocorreu de modo concomitante com a permanência em sala durante o recreio, o SIB permaneceu em uma frequência baixa. Na reversão à linha de base, ocorreu aumento do SIB, que permaneceu em frequência alta e oscilante, mesmo após a reintrodução do recreio em sala.

Na sexta sessão, registrou-se um pico atípico na frequência de SIB, que aumentou para mais do que o dobro de respostas em relação à linha de base. Ao serem investigadas variáveis que pudessem estar relacionadas a esse aumento, constatou-se que, três dias antes, o participante havia sido transferido de casa, devido a uma obra de reforma. Tal mudança na rotina doméstica foi acompanhada de aumento na frequência de SIB também no novo ambiente doméstico. Sabe-se que pessoas com transtorno do espectro do autismo apresentam aderência inflexível e excessiva a rotinas e costumam sofrer durante o processo de adaptação a alterações bruscas nessas rotinas (Associação Psiquiátrica Americana, 2013), o que pode explicar a mudança do comportamento do participante nesse período.

Quando foi introduzido o procedimento de treino de mando em substituição às atividades anteriormente realizadas, observou-se novamente redução do SIB. Na Figura 3, observam-se as porcentagens de respostas independentes do participante a cada sessão do treino de mando.

\section{Follow up}

Após 30 dias do fim dos registros dos procedimentos de intervenção, foram realizadas quatro sessões videogravadas de follow-up no ambiente escolar, de duração de 40 minutos cada uma. Os registros contemplaram os 10 minutos finais do recreio e 30 minutos subsequentes em sala, durante períodos de atividades, intercalados com a permanência na cadeira do lazer. A professora foi orientada a dar continuidade aos procedimentos de treino de mando e NCR durante o intervalo que antecedeu o follow-up. Foi mantido também o recreio em sala, com livre acesso ao colchonete.

A frequência de SIB observada durante o followup está representada na Figura 2. Na Figura 3 estão representadas as respostas independentes de mando ocorridas nas sessões de follow-up. A concordância entre observadores para as respostas independentes de mando nas sessões de follow-up também foi calculada por meio do método de concordância total, como nas etapas anteriores, obtendo-se média de concordância de 97,8\%.

Na primeira e na quarta sessões de follow-up, não houve ocorrência de SIB. Na terceira sessão, houve uma única ocorrência e na segunda sessão foram 16 ocorrências. 


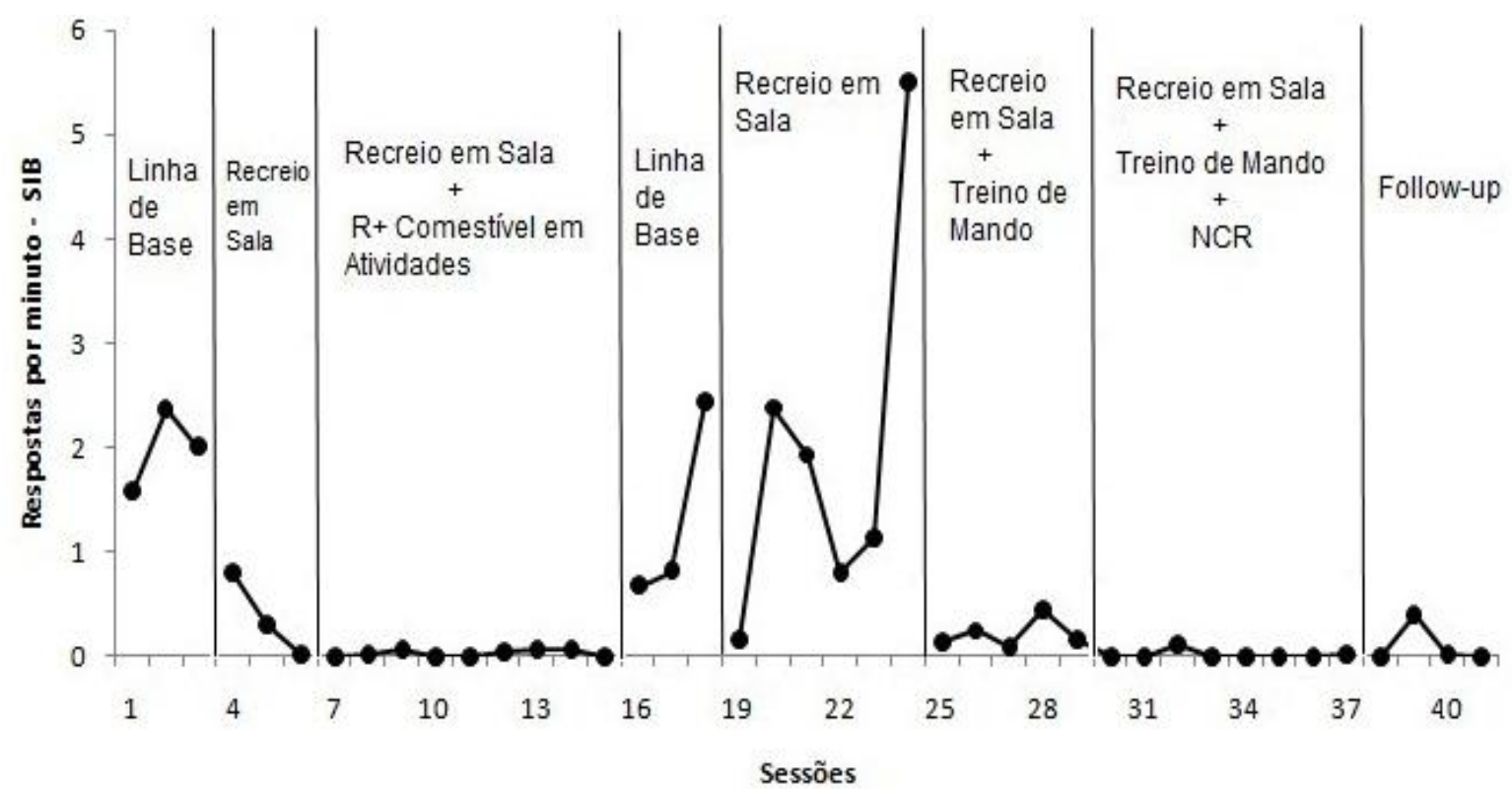

Figura 2. Taxa média de respostas de SIB (R/minuto) ao longo das sessões de intervenção realizadas no ambiente escolar e no followup, realizado um mês depois.

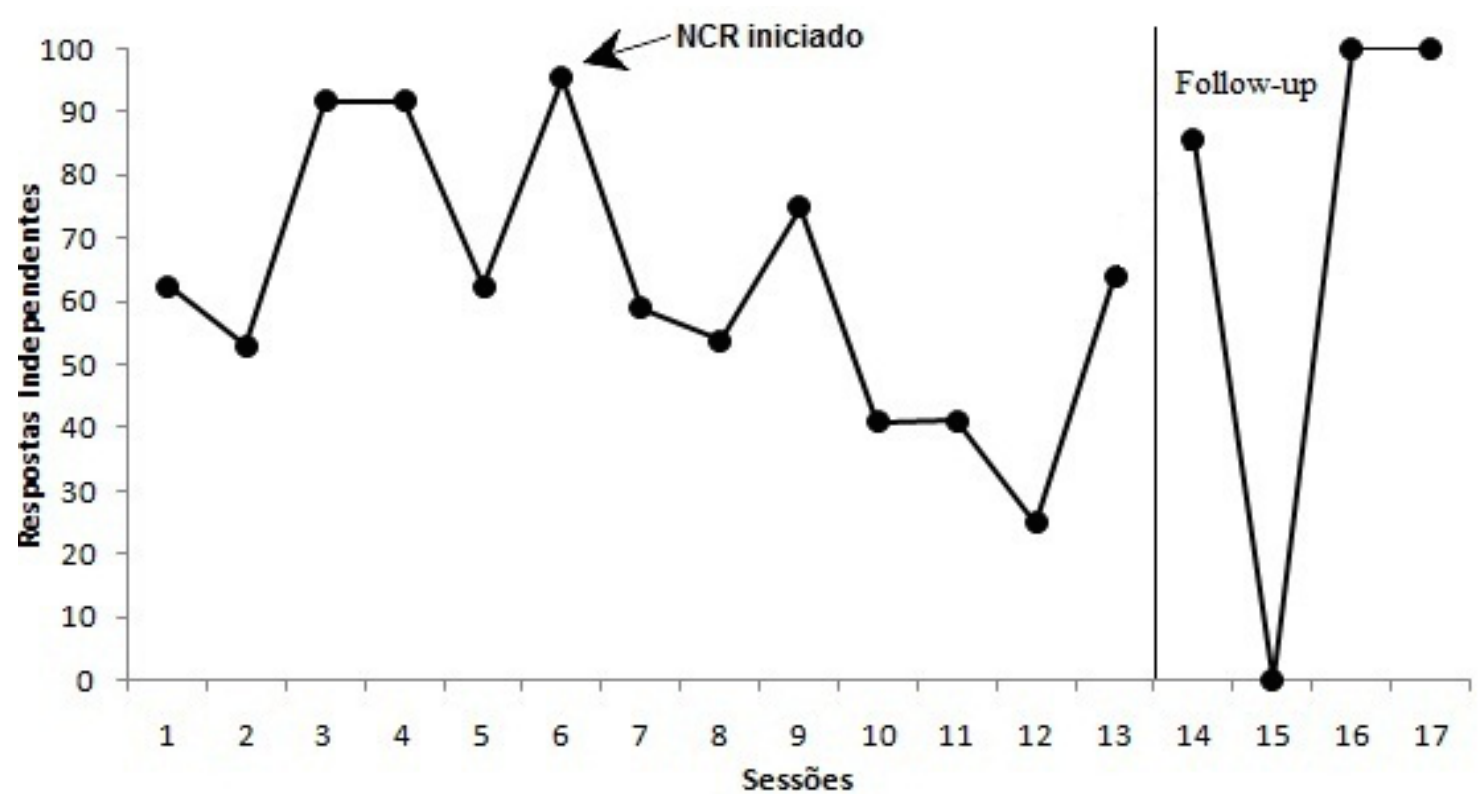

Figura 3. Porcentagem de respostas independentes de mando ao longo das sessões de intervenção e em quarto sessões de follow-up.

Observou-se também que, nessa segunda sessão, não ocorreram respostas independentes de mando, enquanto em todas as demais sessões a frequência de mandos independentes foi alta, chegando a $100 \%$ na terceira e quarta sessões. Na segunda sessão, foi observado que o participante não estava sensível aos reforçadores comestíveis, mas, ainda assim, a professora mantinha as tentativas discretas e direcionamentos físicos, estabelecendo relações que podem ter se tornado aversivas. Essa observação sugere que seria importante o planejamento de atividades diferentes, que pudessem ser realizadas quando o participante não estivesse sensível aos reforçadores comestíveis.

Os relatos da professora e da auxiliar foram de que, durante o período de um mês entre o fim dos registros da intervenção e o início dos registros de follow-up, houve a manutenção de uma freqüência baixa de SIB, que permaneceu em frequência zero durante muitos dos dias letivos. Relataram ainda que não necessitaram, durante o período, recorrer à direção da escola devido a crises de auto-agressão do participante, o que ocorria com frequência antes da intervenção. 


\section{DISCUSSÃO}

Tem sido evidenciada a importância de procedimentos de análise funcional experimental para o embasamento de planejamentos eficazes de intervenções de tratamento baseado em evidências para o SIB (Dixon, Vogel, \& Tarbox, 2012; Sturmey \& Hersen, 2012) em contraposição a estudos que demonstraram a menor eficácia de métodos indiretos ou descritivos de avaliação funcional (Alter, Conroy, Mancil, \& Haydon, 2008; Lang et al., 2009; Pence, Roscoe, Bourret, \& Ahearn, 2009; Sasso et al., 1992; Tarbox et al., 2009; Thompson \& Iwata, 2007). O presente estudo atingiu seu propósito ao demonstrar a efetividade de procedimentos de reforçamento não contingente e treino de mando planejados com base nos resultados de uma análise funcional experimental, visando à redução do SIB em um indivíduo com deficiência severa.

Os dados obtidos na análise funcional experimental conduzida demonstraram que a frequência de SIB foi maior quando demandas foram apresentadas ao participante e o SIB produzia cessação imediata dessas demandas. A constatação da função do SIB para o participante teve fundamental importância para o arranjo de novas condições no ambiente escolar, onde a ocorrência do SIB era alta.

O uso de reforçador comestível durante a realização de atividades visava produzir maior engajamento do participante e redução da aversividade nas interações na mesa de atividades. Em sua rotina, o participante tinha as mãos direcionadas fisicamente para realizar transferências de objetos de um pote para outro e não apresentava qualquer resposta independente, constantemente puxando os braços para trás, por exemplo, e demonstrando recusa em atender às dicas físicas da professora.

O fornecimento de reforçadores positivos no tratamento pode abolir propriedades aversivas das tarefas e, consequentemente, reduzir a emissão do comportamento-problema (Geiger, Carr, \& LeBlanc, 2010). Lalli et al., (1999) consideraram, por exemplo, que o fornecimento de reforçadores positivos para comportamentos colaborativos pode ser mais efetivo na redução de comportamentos-problema mantidos por reforçamento negativo, do que o fornecimento de pausas na tarefa contingentes a comportamentos colaborativos, ainda que o comportamento-problema continuasse a produzir fuga das tarefas.

A frequência de SIB, que já havia reduzido após a permanência em sala durante o recreio, manteve-se em níveis muito baixos em relação à linha de base, durante toda a fase em que a maçã foi usada como reforçador. Entretanto, as atividades de transferência de objetos não pareciam instalar no repertório do participante comportamentos que pudessem vir a produzir consequências que lhe fossem funcionais em situações naturais na interação com os outros. Assim, visando o ensino de um repertório que fosse funcionalmente mais relevante para o participante, de modo que ele pudesse exercer maior controle sobre o ambiente social, as atividades de transferência de objetos foram substituídas pelo treino de mando. Os dados mostraram que, embora o repertório de L. fosse bastante limitado, ele aprendeu a emitir mandos e este comportamento se manteve durante o follow up, evidenciando um dado da literatura de que a aprendizagem de respostas relevantes para a vida diária pode contribuir para a redução de SIB (Chambers \& Rehfeldt, 2003; Frea, Arnold, \& Vittimberga, 2001; Kelley, Shillingsburg, Castro, Adisson, \& LaRue, 2007).

Quando foi introduzido o treino de mando, devido à baixa acuidade visual do participante, foram utilizadas muitas dicas físicas completas nas duas sessões iniciais, com muitas respostas direcionadas fisicamente. A partir da sexta sessão de treino de mando, foi implementado também o procedimento de reforçamento não-contingente (NCR), no qual os reforçadores comestíveis eram disponibilizados ao participante em um esquema de tempo variável (VT), durante os períodos em que ele permanecia sentado na cadeira do lazer, entre um treino e outro. Ingvarsson, Kahng e Hausman (2008) relataram que o reforçamento não-contingente com um item comestível foi suficiente para aumentar comportamentos colaborativos e reduzir a taxa de comportamento-problema, sem a utilização de extinção de fuga, em um contexto de demanda. Uma hipótese discutida na literatura para o sucesso no uso do procedimento de NCR é a provável diminuição da motivação para emissão de comportamentos problema para obter reforço, uma vez que reforçadores estão livremente disponíveis (Carr et al., 2000). Neste estudo, o uso do NCR, somado ao aprendizado de emitir respostas independentes de mando, pode ter sido crítico para a redução aparentemente estável da frequência de emissão de SIB.

Um aspecto discutido por Carr et al. (2000) é a necessidade de se explorar a transferência da aplicabilidade do NCR de ambientes controlados para situações naturais. Assim, dar continuidade ao procedimento de NCR em instituições como a APAE, ao longo de todo um ano letivo, exigiria o esvanecimento (fading out) do fornecimento de reforçadores no esquema $\mathrm{NCR}$, até que se alcançassem esquemas viáveis de serem mantidos na rotina institucional por períodos mais longos, mantendo-se, no entanto, o SIB em níveis baixos de ocorrência. Futuros estudos podem contemplar um acompanhamento mais longo de participantes, envolvendo o esvanecimento do fornecimento de reforçadores em esquemas NCR.

Apesar das vantagens discutidas da combinação dos procedimento de NCR com treino de mando, é possível que o NCR tenha contribuído para a saciação do participante em algumas das sessões do treino de mando, diminuindo assim a efetividade dos estímulos reforçadores comestíveis utilizados, o que contribuiria para explicar a diminuição de respostas independentes nessa fase. Outra hipótese, que pode ser levantada, é que o fornecimento de reforçadores no esquema NCR durante a permanência na cadeira do lazer pode ter criado um esquema concorrente, no qual o custo de resposta para obtenção de reforçadores era maior durante a realização de atividades, enquanto não 
era necessário esforço algum para obtenção do reforço na cadeira do lazer.

O follow-up no presente estudo foi feito após o período de um mês, o que talvez seja pouco tempo para que seja avaliada uma maior consistência na continuidade dos resultados, ou mesmo a viabilidade da manutenção dos procedimentos por períodos mais longos na instituição. Pode ser importante que dados sejam coletados de modo longitudinal, em parceria com equipes clínicas que atuam em instituições. Na instituição em que o presente estudo foi realizado, geralmente os procedimentos de ensino são modificados após o início de um novo ano letivo, com mudança de professores e auxiliares, de modo que um acompanhamento longitudinal parece relevante para a aplicação de procedimentos deste tipo.

$\mathrm{O}$ estudo pode contribuir, no âmbito nacional, para fortalecer o já amplo corpo de evidências que embasa a adoção de procedimentos de análise funcional experimental para o planejamento de tratamentos do SIB. Futuras pesquisas podem envolver estudos com mais de um participante, delineamentos mais complexos, como linha de base múltipla, bem como variações de procedimentos de análise funcional experimental, de acordo com as especificidades dos contextos de aplicação.

\section{REFERÊNCIAS}

Alter, P. J., Conroy, M. A., Mancil, G. R., \& Haydon, T. (2008). A comparison of functional behavior assessment methodologies with young children: Descriptive methods and functional analysis. Journal of Behavioral Education, 17, 200-219.

Associação Psiquiátrica Americana. (2013). Manual Diagnóstico e Estatístico de Transtornos Mentais (5 ed.), Arlington, VA: American Psychiatric Publishing.

Baghdadli, A., Pascal, C., Grisi, S., \& Aussilloux, C. (2003). Risk factors for self-injurious behaviours among 222 young children with autistic disorders. Journal of Intellectual Disability Research, 47, 622627.

Barros, R. S. (2003). Uma introdução ao comportamento verbal. Revista Brasileira de Terapia Comportamental e Cognitiva, 5, 73-82.

Broadstock, M., \& Lethaby, A. (2008). The effectiveness of applied behaviour analysis interventions for people with autism spectrum disorder. New Zealand Guidelines Group. Systematic Review. Wellington.

Carr, E. G. (1977). The motivation of self-injurious behavior: A review of some hypotheses. Psychological Bulletin, 84, 800-816.

Carr, J. E., Coriaty, S., Wilder, D. A., Gaunt, B. T., Dozier, C. L., Britton, L. N., Avina, C., \& Reed, C. L. (2000). A review of "noncontinget" reinforcement as treatment for the aberrant behavior of individuals with development disabilities. Research in Developmental Disabilities, 21, 377-391.

Ceppi, B., \& Benvenuti, M. (2011). Análise funcional do comportamento autolesivo. Revista de Psiquiatria Clínica (USP), 38(6), 247-253.

Chambers, M., \& Rehfeldt, R. A. (2003). Assessing the acquisition and generalization of two mands form with adults with severe developmental disabilities. Research in Developmental Disability, 24, 265-280.

Dixon, D. R., Vogel, T., \& Tarbox, J. (2012). A brief history of functional analysis and applied behavior analysis. Em J. L. Matson (Org.), Functional Assesssment for Challenging Behaviors (pp. 3-24). Autism and Child Psychopathology Series. NY: Springer.

Frea, W. D., Arnold, C. L., \& Vittimberga, G. L. (2001). A demonstration of the effects of augmentative communication on the extreme aggressive behavior of a child with autism within an integrated preschool setting. Journal of Positive Behaviour Interventions, 3, 194-198.

Garcia, M. V. F., \& Gomes, C. G. S. (2012). Comportamentos autolesivos e desenvolvimento atípico: uma revisão e análise crítica de procedimentos analítico-comportamentais de avaliação e tratamento no período entre 1959 e 2010. Trabalho de conclusão de curso. Belo Horizonte, MG: Faculdade de Ciências Biológicas e da Saúde do Centro Universitário Newton Paiva.

Geiger, K. B., Carr, J. E., \& LeBlanc, L. A. (2010). Function-based treatments for escape-maintained problem behavior: A treatment-selection model for practicing behavior analysts. Behavior Analysis in Practice, 3, 22-32.

Sturmey, P., \& Hersen, M. (2012). Handbook of evidencebased practice in clinical psychology, child and adolescent disorders. NY: John Wiley \& Sons.

Ingvarsson, E. T., Kahng, S., \& Hausman, N. L. (2008). Some effects of noncontingent positive reinforcement on multiply controlled problem behavior and compliance in a demand context. Journal of Applied Behavior Analysis, 41, 435-440.

Iwata, B. A., Dorsey, M. F., Slifer, K. J., Bauman, K. E., \& Richman, G. S. (1982). Toward a functional analysis of self-injury. Analysis and Intervention in Developmental Disabilities, 2, 3-20. (Reimpresso em 1994: Journal of Applied Behavior Analysis, 27, $197-$ 209).

Iwata, B. A., Pace, G. M., Dorsey, M. F., Zarcone, J. R., Vollmer, T. R., Smith, R. G., Rodgers, T. A., Lerman, D. C., Shore, B. A., \& Mazalesk, J. L. (1994). The functions of self-injurious behavior: An experimentalepidemiological analysis. Journal of Applied Behavior Analysis, 27, 215-40.

Iwata, B. A., Wallace, M. D., Kahng, S., Lindberg, J. S., Roscoe, E. M., Conners, J., et al. (2000). Skill acquisition in the implementation of functional analysis methodology. Journal of Applied Behavior Analysis, 33, 181-194.

Kahng, S., Iwata, B. A, \& Lewin, A. B. (2002). The impact of functional assessment on the treatment of self-injurious behavior. Em S. R. Schroeder, M. L. Oster-Granite, \& T. Thompson (Eds.). Self-injurious behavior: Gene-brain-behavior relationships (pp. 93103). Washington, DC: American Psychological Association. 
Kelley, M. E., Shillingsburg, M. A., Castro, M. J., Adisson, L. R., \& LaRue, R. H. (2007). Further evaluation of emerging speech in children with developmental disabilities: Training verbal behavior. Journal of Applied Behavior Analysis, 40, 431-445.

Lalli, J. S., Casey, S. D., \& Kates, K. (1997). Noncontingent reinforcement as treatment for severe problem behavior: Some procedural variations. Journal of Applied Behavior Analysis, 30, 127-137.

Lalli, J. S., Vollmer, T. R., Progar, P.R., Wright, C., Borrero, J., Daniel. D., Barthold, C. H., Tocco, K., \& May, W. (1999). Competition between positive and negative reinforcement in the treatment of escape behavior. Journal of Applied Behavior Analysis, 32, 285-96.

Lang, R., O'Reilly, M., Lancioni, G., Rispoli, M., Machalicek, W., \& Chan, J. M. (2009). Discrepancy in functional analysis results across two settings: Implications for intervention design. Journal of Applied Behavior Analysis, 42, 393-397.

Lerman, D. C., \& Iwata, B. A. (1993). Descriptive and experimental analysis of variables maintaining selfinjurious behavior. Journal of Applied Behavior Analysis, 26, 293-319.

Matson, J. L., \& Lo Vullo, S. V. (2008). A review of behavioral treatments for self-injurious behaviors of persons with autism spectrum disorders. Behavior Modification, 32, 61-76.

Murphy, C., Barnes-Holmes, D., \& Barnes-Holmes, Y. (2005). Derived manding in children with autism: Synthesizing Skinner's verbal behavior with Relational Frame Theory. Journal of Applied Behavior Analysis, $38,445-462$.

Payne, S. W., \& Dozier, C. L. (2013). Positive reinforcement as treatment for problem behavior maintained by negative reinforcement. Journal of Applied Behavior Analysis, 46, 699-703.

Pence, S. T., Roscoe, E. M., Bourret, J. C., \& Ahearn, W. H. (2009). Relative contributions of three descriptive methods: Implications for behavioral assessment. Journal of Applied Behavior Analysis, 42, 425-446.

Rosales, R., \& Rehfeldt, R. A. (2007). Contriving transitive conditioned establishing operations to establish derived manding skills in adults with severe developmental disabilities. Journal of Applied Behavior Analysis, 40, 105-121.

Saloviita, T. (2000). The structure and correlates of selfinjurious behavior in an institutional setting. Research in Developmental Disabilities, 21, 501-511.

Sasso, G., Reimers, T. M., Cooper, L. J., Wacker, D., Berg, W., Steege, M., \& Allaire, A. (1992). Use of descriptive and experimental analyses to identify the functional properties of aberrant behavior in school settings. Journal of Applied Behavior Analysis, 25, 809-821.

Skinner, B.F. (1957). Verbal behavior. New York: Appleton-Century-Crofts.

Sturmey, P., \& Hersen, M. (2012). Handbook of evidencebased practice in clinical psychology. Hoboken, N.J: John Wiley \& Sons.
Tarbox, J., Wilke, A. E., Najdowski, A. C., Findel-Pyles, R. S., Balasanyan, S., Caveney, A. C., Chilingaryan, V., King, D. M., Niehoff, S. M., Slease, K., \& Tia, B. (2009). Comparing indirect, descriptive, and experimental functional assessments of challenging behavior in children with autism. Journal of Development and Physical Disabilities, 21, 493-514.

Tate, B. G., \& Baroff, G. S. (1966). Aversive control of self-injurious behavior in a psychotic boy. Behaviour Research and Therapy, 4, 281-287.

Thompson R. H., \& Iwata, B. A. (2007). A comparison of outcomes from descriptive and functional analyses of problem behavior. Journal of Applied Behavior Analysis, 40, 333-338.

Troconis, C. (2011). The effects of manipulating conditioned establishing operations on the acquisition of mands in children with autism spectrum disorders. Master Thesis. Tampa, FL.: Graduate School of University of South Florida.

Gutierrez Jr., A., Vollmer, T. R., Dozier, C. L., Borrero, J. C., Rapp, J. T., Bourret, J. C., \& Gadaire, D. (2007). Manipulating establishing operations to verify and establish stimulus control during mand training. Journal of Applied Behavior Analysis, 40, 645-658.

Winett, R. A., Winkler, R. C. (1972). Current behavior modification in the classroom: Be still, be quiet, be docile. Journal of Applied Behavior Analysis, 5, 499 504.

Submetido em 13/11/2015 Aceito em 01/07/2016 